\title{
Análisis descriptivo de los centros al área como indicador de rendimiento en fútbol.
}

Descriptive analysis of the crosses to the box as a performance indicator in football

\section{Autor: Alfonso Cano Sáez}

\author{
Director: Luis Fradua Uriondo
}
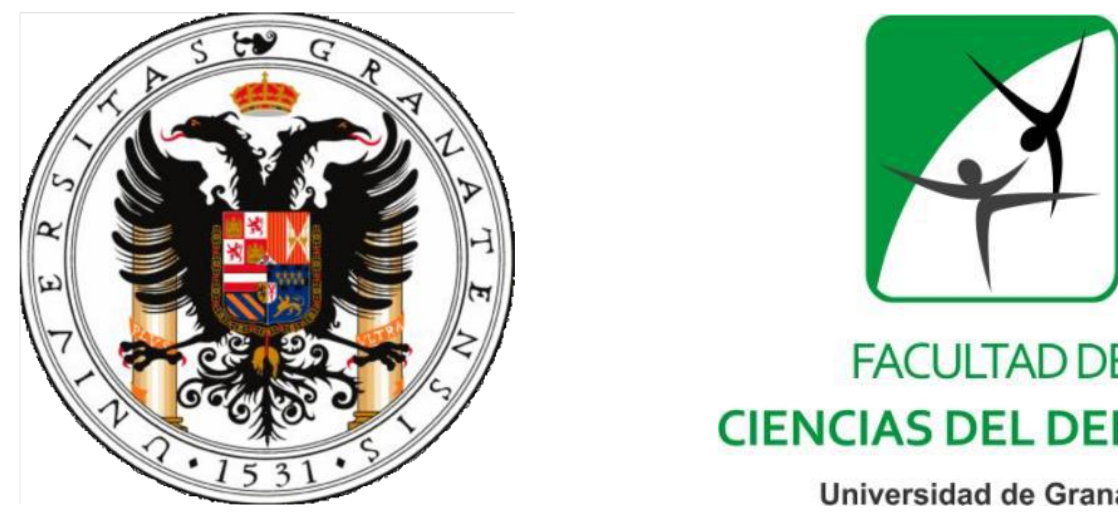

FACULTADDE

CIENCIAS DEL DEPORTE

Universidad de Granada

Máster de Investigación en Actividad Física y Deporte Trabajo Fin de Máster

Departamento de Actividad física y Deportiva

Facultad de Ciencias del Deporte

Universidad de Granada 
Índice

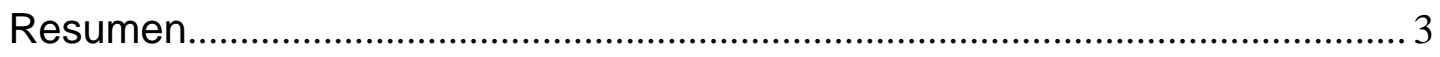

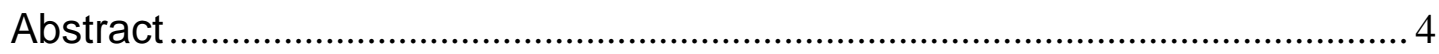

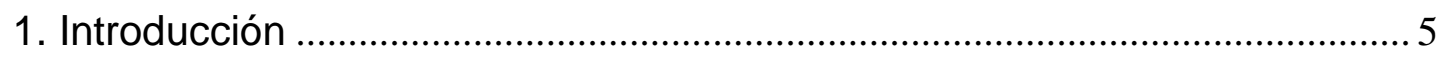

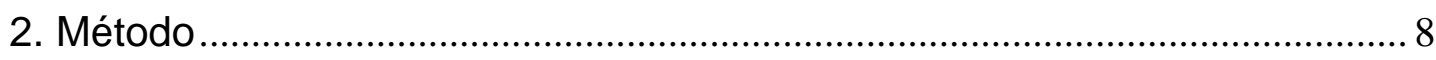

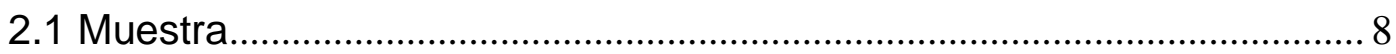

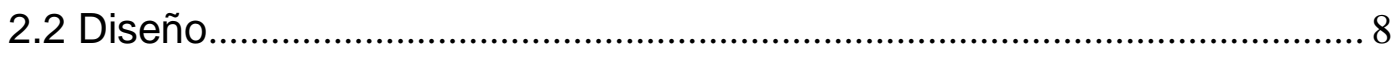

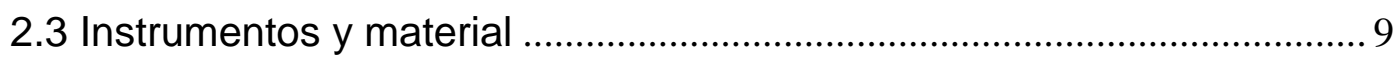

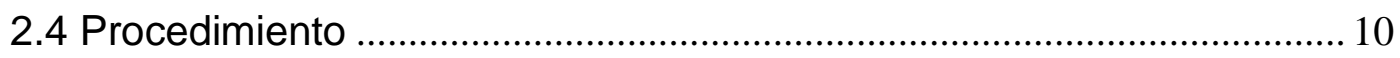

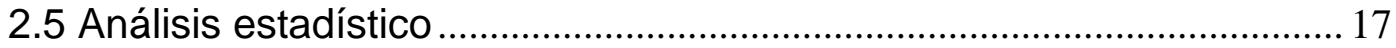

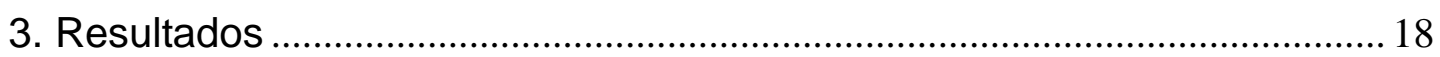

3.1 Variables contextuales del centro por banda......................................... 18

3.2 Variables previas al centro por banda.................................................. 19

3.3 Variables durante y posteriores al centro ......................................... 20

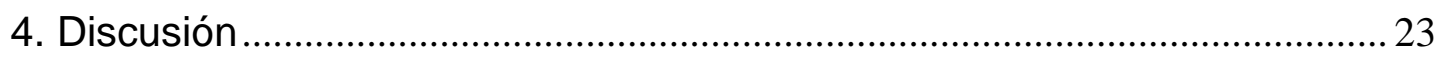

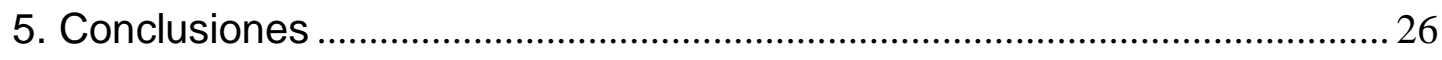

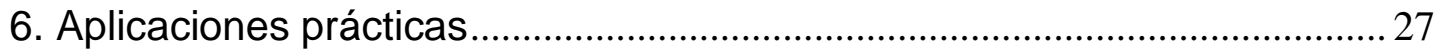

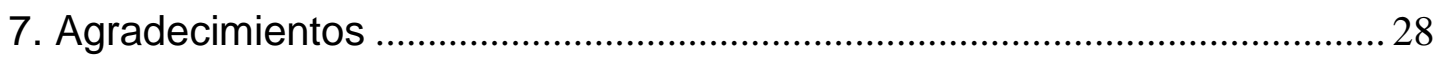

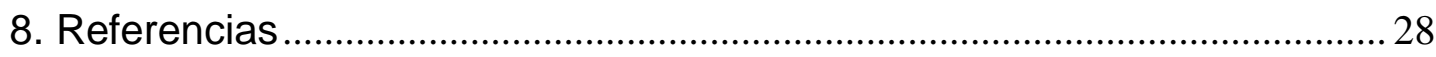




\section{Resumen}

Con el presente estudio se pretendió describir los centros realizados por banda en el futbol de alto nivel; analizar los centros por banda como indicador de rendimiento y proponer aplicaciones prácticas para el entrenamiento de los centros por banda. Se analizaron 32 partidos de la Liga de Futbol Profesional y la Premier League entre las temporadas 2006/2007 y 2010/2011, mediante el sistema de AMISCO PRO®, uno de los más potentes en la actualidad para la recogida de información real en partidos de alto nivel. Se realizó un proceso de categorización de las variables elegidas para el cual se obtuvo un valor Kappa superior a 0,75. Los resultados de este estudio mostraron un $62,4 \%$ de centros de los equipos locales, sobre el total realizado. También pudimos observar una alta participación de jugadores de primera línea, los laterales con un $42,6 \%$ de los centros. Los ataques posicionales duplicaron porcentualmente a los realizados tras robo y los pases al espacio realizados antes del centro fueron los más numerosos $(50,4 \%)$. El presente estudio contribuye a establecer una visión sobre los elementos previos a un centro, a través de la cual se podrá modificar el establecimiento del modelo de juego y las situaciones de entrenamiento con el fin de aumentar el rendimiento.

Palabras clave: análisis del juego, análisis del rendimiento, fútbol, centros 


\begin{abstract}
The present study aims to describe the crosses made by high-level football; analyzing crosses as a performance indicator and to propose practical applications for training crosses. We analized 32 games of the Liga de Fútbol Profesional and the Premier League seasons between 2006/2007 and 2010/2011, through the system of AMISCO $\mathrm{PRO} B$, one of the most powerful today for collecting actual information in high-analyzed matches level. A process of categorization of the variables chosen for which a Kappa value above 0.75 was obtained. The results of this study showed $62.4 \%$ of centers of local teams, the total realized. We also saw a high turnout of players in front, side with $42.6 \%$ of the centers. Positional attacks doubled in percentage terms to those made after theft and passes into space made before the center were the most numerous $(50.4 \%)$. This study contributes to establish a vision on pre-center elements, through which can modify the establishment model playing and training situations in order to increase performance.
\end{abstract}

Keywords: match analysis, performance indicator, football, soccer, crosses 


\section{Introducción}

En fútbol, el rendimiento ha sido definido como la interacción eficaz de los diferentes elementos técnicos, tácticos, mentales (Carling, Reilly, \& Williams, 2008) y fisiológicos (Drust, Atkinson, \& Reilly, 2007). La investigación científica en el ámbito del rendimiento técnico-táctico, estuvo limitada por la falta de información en revistas científicas, (M. Hughes \& I. M. Franks, 2004), hasta la década de los noventa, en la cual, a través de la creación de la ISPAS (International Society of Performance Analysis of Sport), la edición de revistas especializadas (International Journal of Performance Analysis in Sport; Journal of Quantitative Analysis in Sports) y la introducción del Congreso Mundial sobre Análisis Notacional, se ganaron un sitio en la literatura científica (Sarmento et al., 2014).

El análisis del rendimiento, se originó específicamente en los deportes de equipo, en EEUU con el fútbol americano y el baloncesto (Carling, Williams, \& Reilly, 2005; M. Hughes \& I. Franks, 2004), los deportes de raqueta también adaptaron este enfoque antes que el fútbol (Carling et al., 2005). Sin embargo, en las últimas 3 décadas, ha crecido notablemente el desarrollo de sistemas de análisis de rendimiento, y las investigaciones de análisis del rendimiento específicamente para el fútbol (Mike Hughes \& Franks, 2005), al igual que ha aumentado el número de citaciones relacionadas en este ámbito para nuestra mejor comprensión de los requisitos técnicos, tácticos y físicos del fútbol (Bangsbo, Mohr, \& Krustrup, 2006; Bradley et al., 2009; Mohr, Krustrup, \& Bangsbo, 2003).

En contraste con los primeros estudios sobre análisis del partido donde el método de recogida de datos era un papel y un lápiz, para obtener el perfil de actividad de un jugador (Reilly \& Thomas, 1976), en la actualidad, estos métodos han evolucionado gracias a las recientes mejoras tecnológicas (software de análisis de juego, tecnología de sensores remotos, sistemas de video seguimiento, etc.), los cuales, se han desarrollado junto a modelos estadísticos nuevos (predictivos y estocásticos), para inferir o predecir los resultados del rendimiento en el deporte (Nevill, Atkinson, \& Hughes, 2008), lo cual nos ha aportado durante la última década la capacidad de producir datos que proporcionan información sustancial sobre la descripción individual del rendimiento en los deportes de equipo (Liebermann et al., 2002). Una ventaja de la disponibilidad de los datos existente, es que los investigadores han podido superar las limitaciones en cuanto a muestra, que existían en anteriores publicaciones (Coutts, 2014).

En consecuencia, ha habido un aumento de los estudios en el fútbol que analizan cuestiones como la evolución temporal del partido, los perfiles de los jugadores, comparaciones entre las exigencias del partido de diferentes ligas, junto con el impacto de los enfoques tácticos y otros factores contextuales como el nivel del rival, el marcador o las exigencias del partido (Sarmento et al., 2014). Todas 
estas variables, influencian, no solo en el desarrollo del juego, sino también las exigencias físicas (Lago, Casais, Dominguez, \& Sampaio, 2010; Rampinini, Coutts, Castagna, Sassi, \& Impellizzeri, 2007), su rendimiento técnico (Taylor, Mellalieu, James, \& Shearer, 2008), el tiempo efectivo de juego (J Castellano, BlancoVillaseñor, \& Alvarez, 2011) o la posesión (Lago, 2009). Últimamente, algunas variables que también son utilizadas como posibles indicadores del rendimiento, son la anchura que ocupan los jugadores en el campo (Bartlett, Button, Robins, DuttMazumder, \& Kennedy, 2012)

Todo este proceso ha mejorado nuestra capacidad de comprender la actividad y las exigencias del futbol.

Los indicadores de rendimiento se definen como la combinación de variables que definen algunos aspectos del rendimiento deportivos, y ayudan a la obtención del éxito deportivo (M. D. Hughes \& Bartlett, 2002). Estos indicadores, constituyen un perfil de rendimiento ideal, que debe estar presente en la actividad para así, poder lograr este rendimiento y puede ser utilizado como una manera de predecir el futuro comportamiento de la actividad deportiva (Jones, Mellalieu, \& James, 2004; O'Donoghue, 2005). Hasta la fecha un pequeño número de estudios han tratado de proporcionar indicadores de rendimiento del equipo a través de la comparación de ganadores y perdedores (Grant, Williams, Reilly, \& Borrie, 1999; Mike Hughes, Churchill, Reilly, Cabri, \& Araújo, 2005; Mike Hughes \& Franks, 2005; M Hughes, Robertson, \& Nicholson, 1988; Jones et al., 2004; Stanhope, 2001). Sin embargo, los patrones de juego de estos estudios muestran resultados relativamente contradictorios.

La literatura existente en el análisis del rendimiento en futbol, sugiere que hay una escasez de investigación sobre los indicadores de rendimiento de los equipos y de los perfiles de los mismos. Sasaki, Nevill, and Reilly (1999) encontró que se realizaron más ocasiones de gol, disparos, disparos parados, disparos a puerta y centros con éxito durante los partidos en casa, en comparación con los partidos fuera. Del mismo modo, Tucker, Mellalieu, James, and Taylor (2005) encontraron que se dieron un mayor número de córner, centros, regates, pases y disparos en los partidos de casa. En cuanto al efecto que produce el jugar dentro o fuera de casa, Lago-Ballesteros and Lago-Peñas (2010) observaron un tamaño del efecto más fuerte en pérdidas de balón en la zona 5.2 y de centros en la zona 4, los equipos ganadores, también tenían un menor número de centros a la zona 4 , lo que significa que el balón llegó zona 5.2, lo que explicaron por el mayor uso de pases en largo y pases que superan las líneas más efectivos (Tenga, Holme, Ronglan, \& Bahr, 2010). Otras explicaciones a este hecho pudieron estar asociadas a más pases laterales de los lados (zonas 5,4 y 5,5 ). 
Sasaki et al. (1999) por ejemplo, analizó los equipos de la primera División de la Liga inglesa en la 96-97 y encontró diferencias significativas en las ocasiones de gol, disparos a puerta, disparos parados, disparos fuera y centros con éxito. Tucker et al. (2005) también encontró en su estudio, que los equipos ingleses realizan un mayor número de córner, centros, regates, pases, disparos durante los partidos de casa, en un examen adicional, los resultados mostraron más éxito en los centros, pases y entradas durante los partidos de casa. Sin embargo, Taylor et al. (2008) encontraron que en los equipos profesionales británicos que estudiaron, los resultados de la mayoría de los comportamientos no fueron influenciados por la localización del partido.

Griffiths (1999) seleccionó los partidos de Francia, que se encontraban en ese momento considerado el mejor equipo internacional en el Mundial. Se extrajo que Francia era capaz de crear significativamente más disparos mientras que también tiene la capacidad de retener la posesión durante largos períodos. Curiosamente Francia también creó significativamente más centros que sus oponentes, lo que sugiere que en el fútbol moderno, el éxito de los equipos viene dado por utilizar ataques por las bandas con más frecuencia que lo extraído de investigaciones anteriores, por ejemplo, M Hughes et al. (1988).

Como hemos observado, son numerosos los autores que estudian los indicadores de rendimiento en futbol, entre los cuales, se ubican los centros, pero ninguno lo ha estudiado de forma concreta como hacemos nosotros. Nuestro interés ha sido estudiar el comportamiento por banda de los equipos de máximo nivel para establecer un rendimiento en ataque. Por lo tanto pretendemos observar si los centros por banda, pueden ser un indicador de rendimiento, para ello los objetivos de este estudio son:

Los objetivos de este estudio son: 1) Describir los centros realizados por banda en el futbol de alto nivel. 2) Analizar los centros por banda como indicador de rendimiento 3) Proponer aplicaciones prácticas para el entrenamiento de los centros por banda. 


\section{Método}

\subsection{Muestra}

En este estudio, se analizaron 32 partidos de la Liga de Futbol Profesional y la Premier League entre las temporadas 2006/2007 y 2010/2011, mediante el sistema de AMISCO PRO®. La muestra recogida, está formada por los centros laterales de las zonas $5 \mathrm{~A} / \mathrm{D}$ y $6 \mathrm{~A} / \mathrm{D}$ hacia las zonas $6 \mathrm{~B}$ o $\mathrm{D}$, tal y como viene representado en la figura 1, que se dan con el balón en juego durante el transcurso del encuentro (fueron excluidos todos los inicios a balón parado). Fueron contabilizados un total de 657 eventos $(n=657)$. La muestra fue representada por un total de 22 equipos y una media de 20,53 centros por partido.

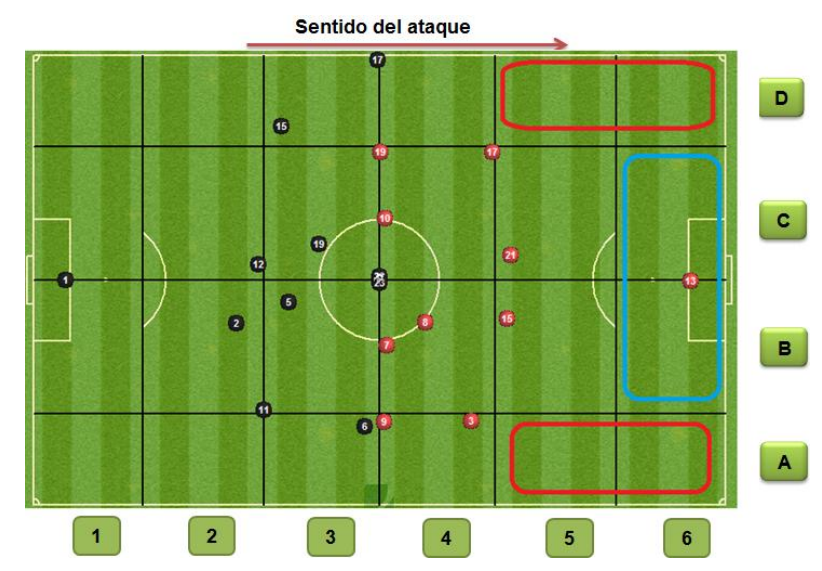

Figura 1: representación de las zonas del campo en las que se producen los centro (rojo) y las zonas en las que se reciben los centros (azul)

\subsection{Diseño}

Se trata de un estudio descriptivo de metodología observacional, ya que tiene un grado de control interno bajo para no desnaturalizar el juego. Sin embargo, cuenta con un grado de perceptividad máximo, ya que se ha realizado mediante observación directa, a través de análisis de vídeos.

Es un diseño de seguimiento, nomotético puntual multidimensional, ya que analizamos diversos partidos en un momento concreto de la temporada, con el fin de generalizar los resultados a la población estudiada mediante el registro de las conductas de diferentes jugadores y equipos, y teniendo en cuenta la multitud de variables a analizar debido a la complejidad y a la alta incertidumbre de este deporte. 


\subsection{Instrumentos y material}

Los partidos de la muestra fueron registrados por el sistema AMISCO $\mathrm{PRO} \AA^{\circ}$, un sistema semiautomático de seguimiento a través de 8 cámaras, que mide en video los movimientos de cada jugador, el árbitro y el balón con una actividad de muestreo de 25 veces por segundo durante todo el partido. El sistema crea una reconstrucción animada en 2 dimensiones de los movimientos del jugador y permite el análisis simultáneo de sus movimientos durante todo el partido. La información de cada partido está almacenada en un DVD y puede ser extraída a través de un software específico (Amisco Viewer) (Figura 2). AMISCO PRO® también denominado Prozone, es uno de los sistemas más potentes en la actualidad para la recogida de información real en partidos de alto nivel. La validez de este sistema ha sido ya confirmada en numerosas investigaciones (Carling, Bloomfield, Nelsen, \& Reilly, 2008; Valter, Adam, Barry, \& Marco, 2006; Zubillaga, Gorospe, Hernandez Mendo, \& Villasenor, 2007). La información aportada, fue sometida a un proceso de categorización para obtener los datos definitivos que fueron posteriormente incluidos en una base de datos de IBM SPSS Statistics 20, para el posterior análisis estadístico.

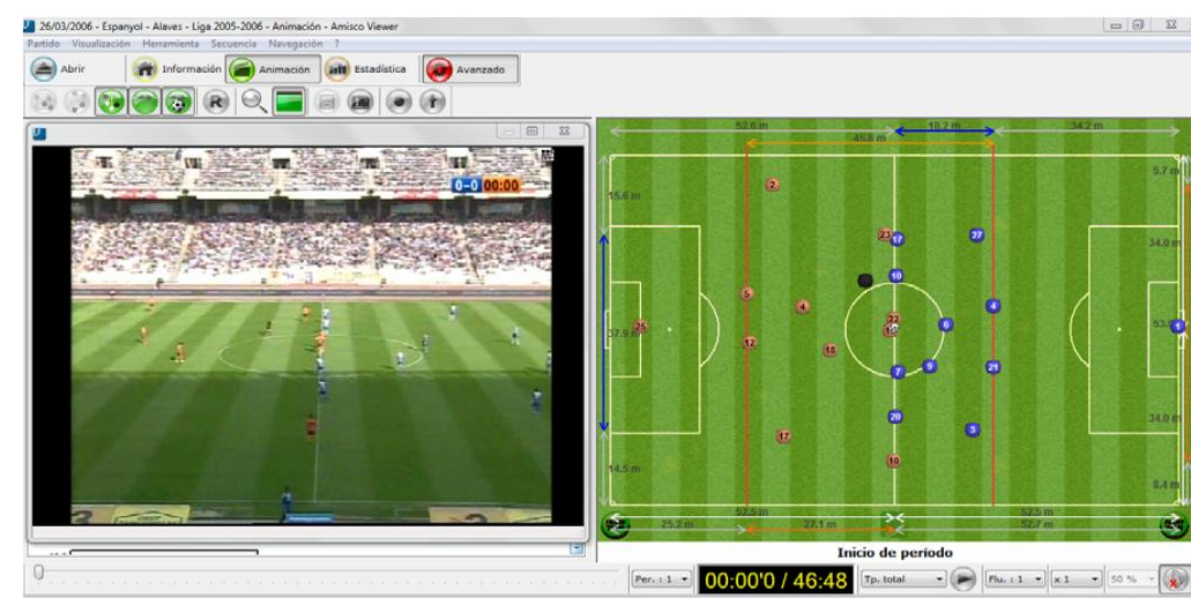

Figura 2: Representación del partido obtenida de Animation Mode AMISCO PRO, Niza, Francia (derecha) y el vídeo sincronizado (izquierda). 


\subsection{Procedimiento}

El presente estudio es un estudio descriptivo en el que se han analizado 25 partidos de fútbol de Primera División de la temporada 2010-2011 y 7 partidos de la Premier League de la temporada 2006/2007. En cada centro se han analizado las variables que podemos observar en la tabla 1 mediante el programa de análisis de juego AMISCO PRO ${ }^{\circledR}$. Realizamos un procedimiento de categorización (ver tabla 1) de los comportamientos observados en los archivos de video. Para ello, ha sido entrenado un sujeto durante un mes en la recogida de datos. Los datos recogidos en cada evento eran registrados en el Programa estadístico SPSS.

Para determinar la fiabilidad del sistema de categorías se llevó a cabo un análisis intra e inter-observador de todas las variables registradas, Después de la recolección de datos dejamos un periodo de cuatro semanas de descanso y el observador volvió a recoger un $10 \%$ de los datos de la muestra de forma aleatoria. Entrenamos a otro observador (Tabachnick \& Fidell, 2013). Como podemos ver en la tabla 2, los valores de Kappa de Cohen (1960) de todos los análisis fueron mayores de 0.75 tomando como referencia los valores propuestos por Fleiss, Levin, and Paik (2013).

\begin{tabular}{|l|l|}
\hline $\begin{array}{l}\text { Tabla 1: categorización de los comportamientos observados en los archivos de } \\
\text { video. Variables extraídas y sus criterios de registro. }\end{array}$ \\
\hline $\begin{array}{l}\text { Situación de } \\
\text { Equipo }\end{array}$ & $\begin{array}{l}\text { Variable: el equipo que realiza el centro juega como local o } \\
\text { visitante. } \\
\text { Criterios del registro: en el momento del centro se categoriza el } \\
\text { evento como local o como visitante, en función de quien sea el } \\
\text { jugador que lo ejecuta. }\end{array}$ \\
\hline Resultado & $\begin{array}{l}\text { Variable: indica si el equipo que centra está perdiendo, } \\
\text { empatando o ganando. } \\
\text { Criterios del registro: en el momento del centro se categoriza el } \\
\text { evento como perdiendo, empatando o ganando, tomando como } \\
\text { referencia el marcador en el momento en que se produce el } \\
\text { evento. }\end{array}$ \\
\hline Jugador \\
centra
\end{tabular}




\begin{tabular}{|c|c|}
\hline & $\begin{array}{l}\text { se realizan cambios, se vuelven a comprobar las posiciones en } \\
\text { los inicios de juego. Estas posiciones son: defensa, } \\
\text { centrocampista o delantero. }\end{array}$ \\
\hline Tipo de ataque & $\begin{array}{l}\text { Variable: observamos si el centro se produce tras un robo de } \\
\text { balón con la correspondiente transición ataque-defensa rápida, o } \\
\text { tras un ataque posicional. } \\
\text { Criterios del registro: los centros vienen precedidos de un } \\
\text { ataque tras un robo o un ataque posicional: } \\
\text { - Un ataque tras robo es cuando un jugador del equipo que } \\
\text { centra se hace con la posesión de la pelota, y en la misma } \\
\text { jugada de forma ininterrumpida, con un numero de pases } \\
\text { inferior a } 3 \text { ( } 3^{\circ} \text { no incluido) realiza el centro. } \\
\text { - Un ataque posicional, es un ataque en el que el equipo que } \\
\text { centra realiza en la posesión previa al centro una serie de } \\
\text { pases mayor a } 3 \text { ( } 3^{\circ} \text { incluido) o en caso de realizar una } \\
\text { secuencia de pases menor, se contabilizara como posicional si } \\
\text { el inicio de la jugada ha sido un saque de banda, esquina o } \\
\text { falta. }\end{array}$ \\
\hline $\begin{array}{l}\text { Posesión } \\
\text { categorizada }\end{array}$ & $\begin{array}{l}\text { Variable: se categorizan las posesiones en función del número } \\
\text { de pases en: posesiones cortas, medias o largas. } \\
\text { Criterios del registro: en cada evento se contabilizaron el } \\
\text { numero de pases realizados previos al centro de forma } \\
\text { ininterrumpida (sin contar el centro). A continuación se realizó un } \\
\text { clúster para categorizar el numero de pases obteniendo tres } \\
\text { bloques diferentes: posesión corta ( } 0 \text { a } 2 \text { pases), media ( } 3 \text { a } 5 \\
\text { pases) o larga ( } 6 \text { o más pases) }\end{array}$ \\
\hline $\begin{array}{l}\text { Circulación } \\
\text { balón }\end{array}$ & $\begin{array}{l}\text { Variable: en función a los sectores por los que circula el balón } \\
\text { durante la posesión del equipo en la jugada del centro, se } \\
\text { establecen: sin circulación, solo banda, medio-banda, banda- } \\
\text { medio-banda o banda a banda } \\
\text { Criterios del registro: se contabilizó la zona en la que se originó } \\
\text { la jugada previa al centro, a partir de ahí se contó cómo cambio } \\
\text { de carril cuando la bola representativa del balón, sobrepaso al } \\
\text { completo las líneas divisorias del terreno de juego. Para que } \\
\text { fuesen contabilizadas como circulaciones, debió de existir un } \\
\text { mínimo de } 1 \text { pase, sino estaría encuadrado dentro de la } \\
\text { categoría sin circulación aunque cambiase de carril. }\end{array}$ \\
\hline
\end{tabular}




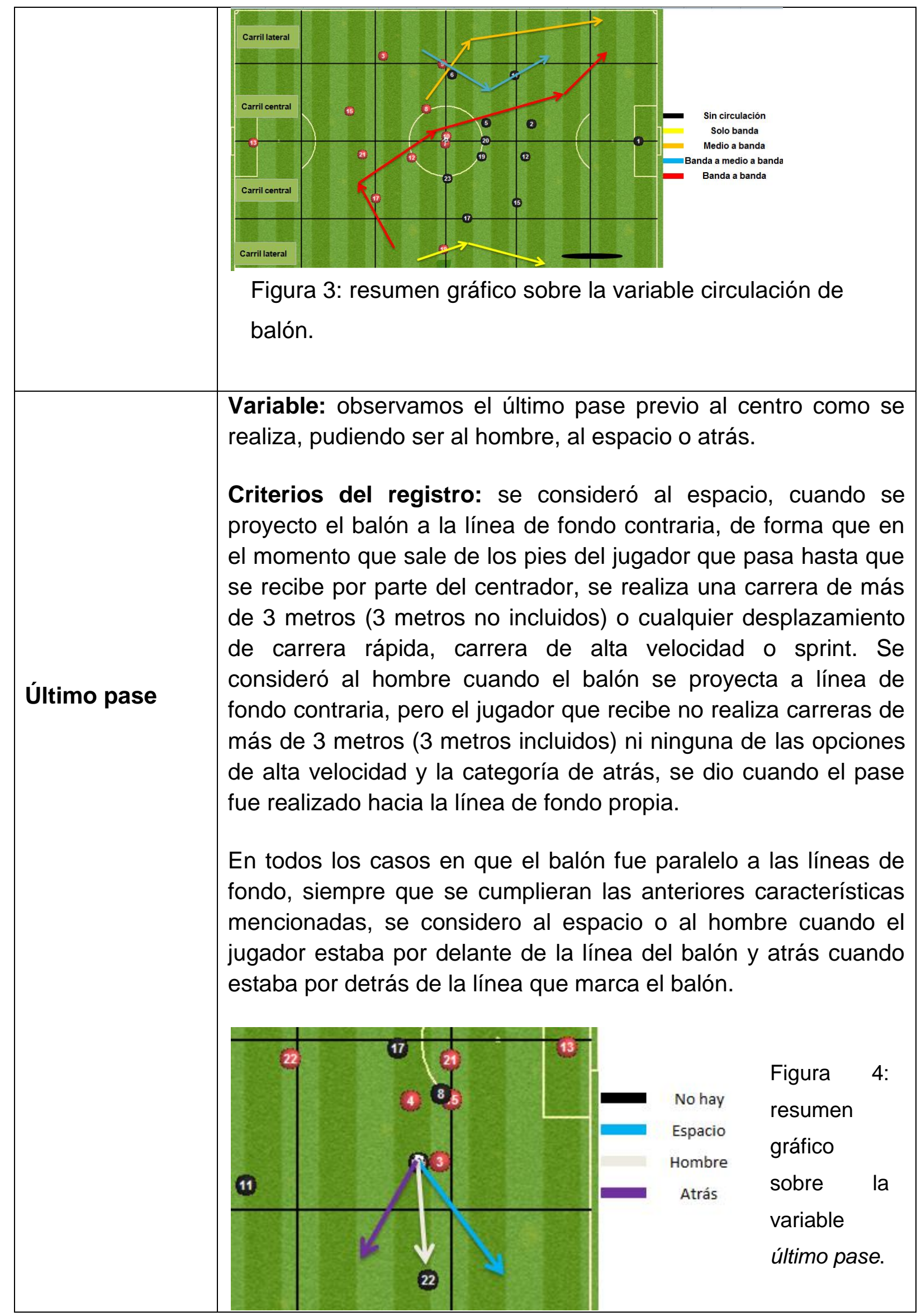




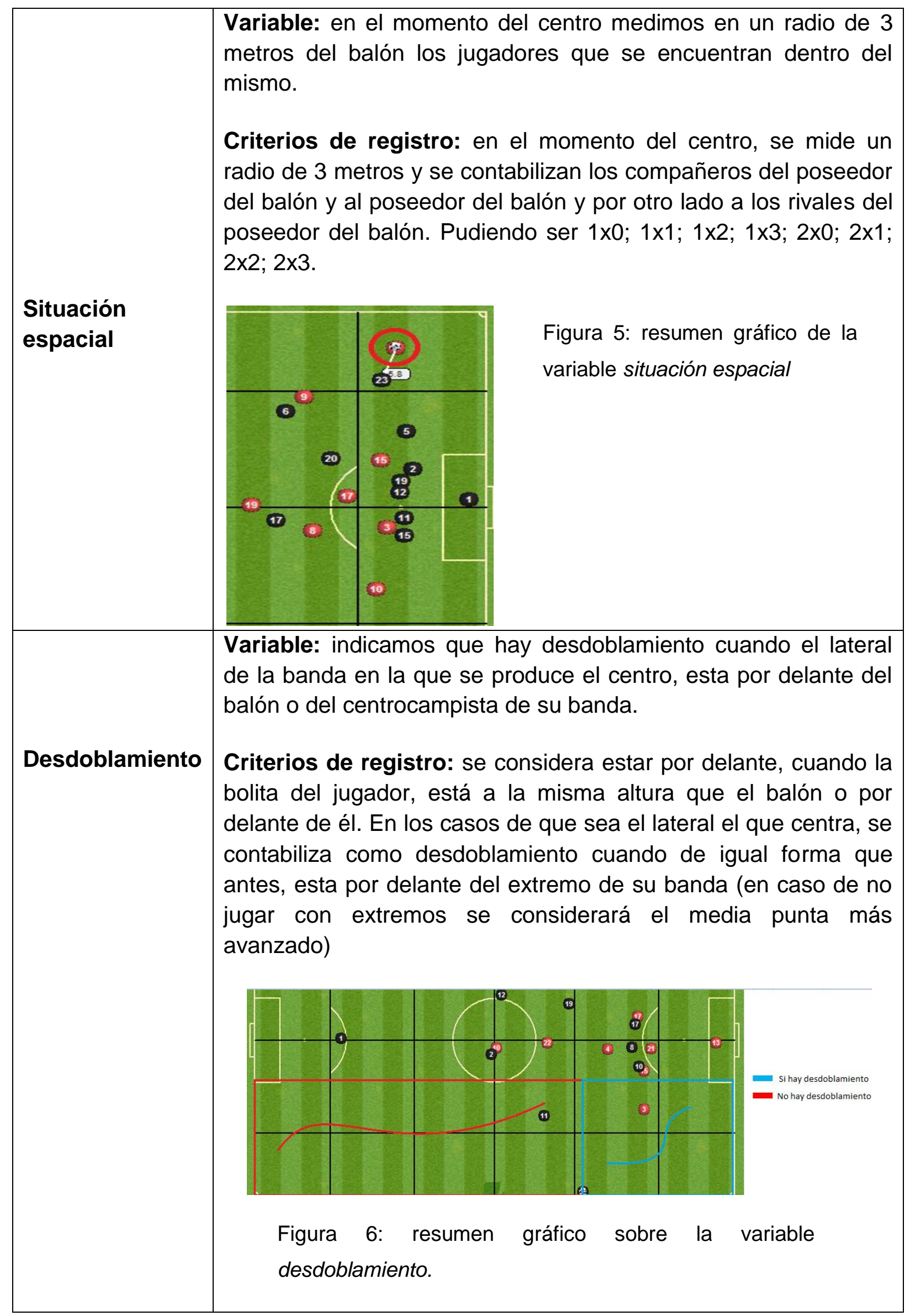




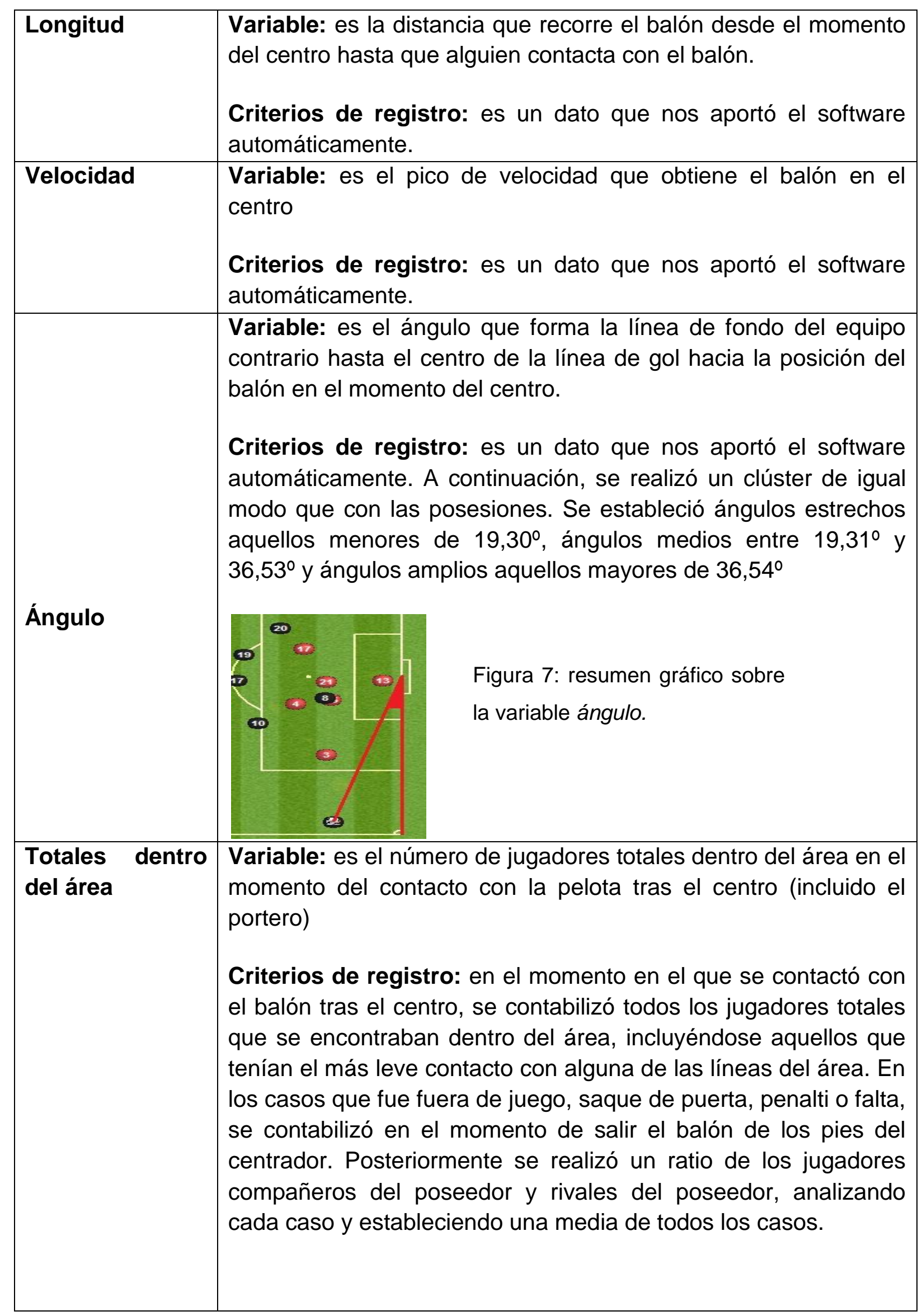




\begin{tabular}{|c|c|}
\hline $\begin{array}{l}\text { Compañeros } \\
\text { dentro del área }\end{array}$ & $\begin{array}{l}\text { Variable: es el número de jugadores atacantes totales dentro del } \\
\text { área en el momento del contacto con la pelota tras el centro } \\
\text { Criterios de registro: se realizó de igual manera que la variable } \\
\text { anterior, pero haciendo hincapié en contabilizar a los } \\
\text { compañeros del poseedor del balón. }\end{array}$ \\
\hline $\begin{array}{l}\text { Rivales dentro } \\
\text { del área }\end{array}$ & $\begin{array}{l}\text { Variable: es el número de jugadores rivales, incluido el portero, } \\
\text { en el momento del contacto con la pelota tras el centro } \\
\text { Criterios de registro: se realizó de igual manera que la variable } \\
\text { anterior, pero haciendo hincapié en contabilizar a los rivales del } \\
\text { poseedor del balón. }\end{array}$ \\
\hline Rendimiento. & 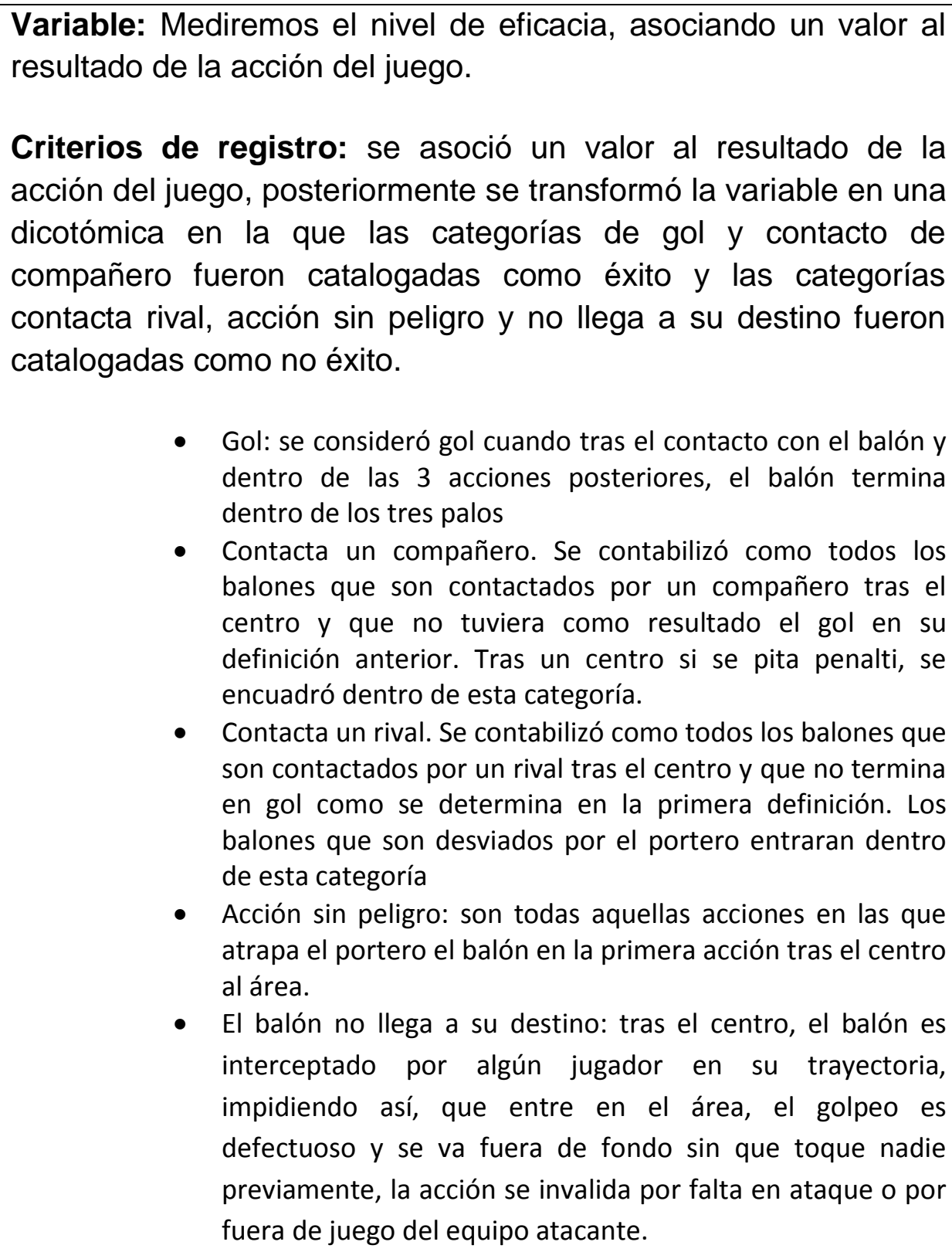 \\
\hline
\end{tabular}




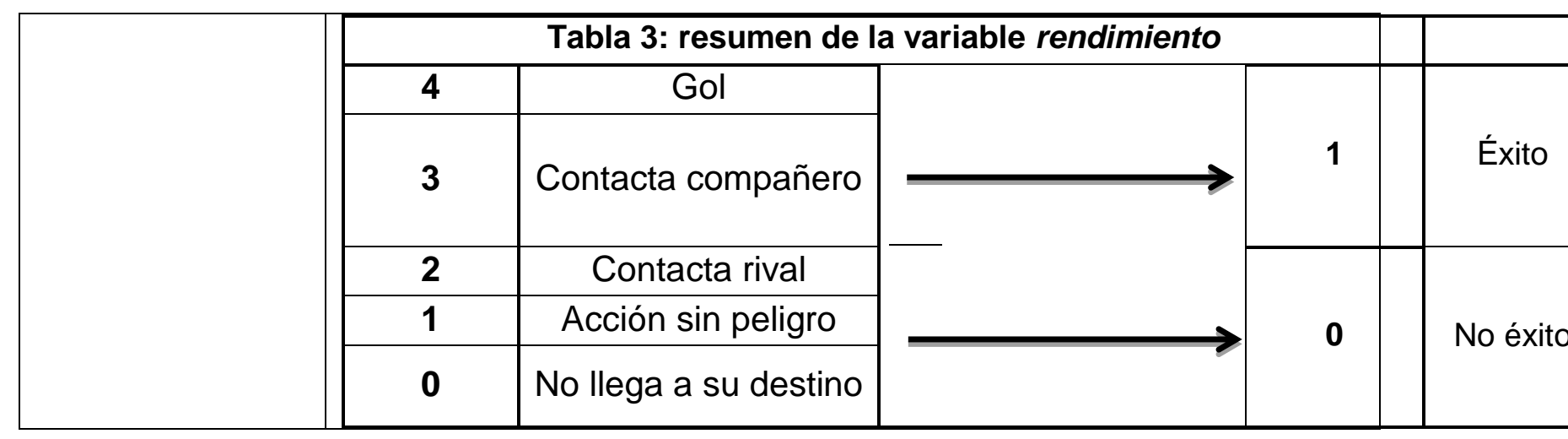

\begin{tabular}{|c|c|c|c|}
\hline \multicolumn{4}{|c|}{ Tabla 2. Fiabilidad Intra-observador e inter-observador } \\
\hline Cohen's Kappa & Intra-observador & Inter-observador & $\begin{array}{c}\text { N } \\
\text { (aleatorio) }\end{array}$ \\
\hline Situación del equipo & 0,871 & 0,935 & 66 \\
\hline $\begin{array}{c}\text { Resultado en función de } \\
\text { la situación del equipo }\end{array}$ & 0,891 & 0,847 & 66 \\
\hline Jugador que centra & 0,921 & 0,948 & 66 \\
\hline Jugador que contacta & 0,901 & 0,92 & 66 \\
\hline Situación tras & 0,958 & 0,979 & 66 \\
\hline Posesión Categorizada & 0,929 & 0,893 & 66 \\
\hline Secuencia & 0,919 & 0,918 & 66 \\
\hline Ultimo pase & 0,952 & 0,976 & 66 \\
\hline Situación numérica & 0,936 & 0,936 & 66 \\
\hline Desdoblamiento & 0,908 & 0,969 & 66 \\
\hline Longitud & 0,969 & 0,969 & 66 \\
\hline Velocidad & 0,985 & 1 & 66 \\
\hline Distancia a gol & 0,938 & 0,954 & 66 \\
\hline Angulo & 0,985 & 1 & 66 \\
\hline Totales dentro del área & 0,914 & 0,931 & 66 \\
\hline Compañeros dentro del & 0,942 & 0,961 & 66 \\
\hline área & 0,924 & 0,943 & 66 \\
\hline Rivales dentro del área & 0,975 & 0,975 & 66 \\
\hline Rendimiento del centro & & & \\
\hline
\end{tabular}




\subsection{Análisis estadístico}

Para el tratamiento descriptivo (univariante) de los datos se empleó un análisis de frecuencias y porcentajes, junto con la respectiva representación gráfica mediante diagramas de sectores y de barras.

Para determinar si existía asociación (bivariante) entre las variables se llevó a cabo la técnica inferencial de correlación no paramétrica, considerando como significativos aquellos resultados con $p<0.05$. Así mismo, se calculó el tamaño del efecto para dichas correlaciones mediante el coeficiente de correlación Rho de Spearman, pero no se aprecio ninguna. Estos análisis se ejecutaron para las variables de Rendimiento, Circulación de balón, Resultado y Tipo de ataque.

Para los análisis univariante y bivariante el instrumento estadístico utilizado fue el paquete estadístico SPSS v.20 para Windows 


\section{Resultados}

A continuación exponemos los datos que hemos encontrado más interesantes después de realizar el análisis descriptivo del presente estudio.

\subsection{Variables contextuales del centro por banda.}

En las siguientes figuras, podemos observar las variables contextuales de los centros por banda. En la figura 8 podemos observar que los equipos que mas centros realizaron en el partido fueron los locales, con un $62,4 \%$, por un $37,6 \%$ de los centros realizados por los visitantes.

Del mismo modo, la figura 9 muestra el porcentaje de casos para la variable Resultado, donde se puede observar que mayoritariamente, los centros son realizados cuando los equipos están en situación de empate, en un $43,4 \%$ de las ocasiones. Los centros realizados cuando el equipo estaba perdiendo fueron un $31,2 \%$ y cuando el equipo estaba ganando, fue un $25,4 \%$.

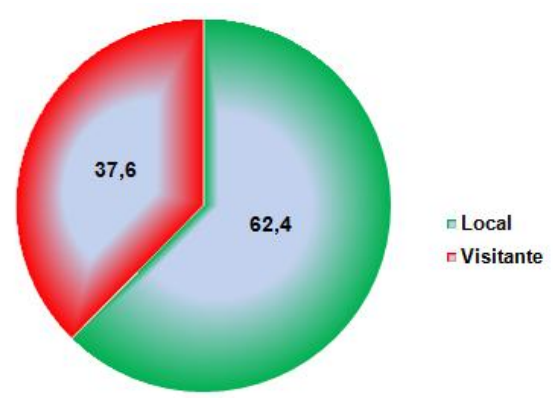

Figura 8:Porcentaje de casos para la variable Situación de equipo $(N=657)$

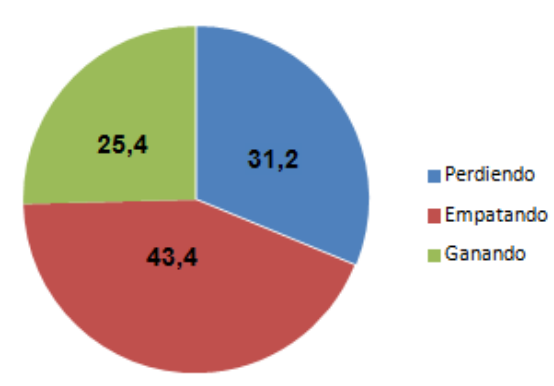

Figura 9: Porcentaje de casos para la variable Resultado $\mathrm{N}=(657)$

En la figura 10, se observan las frecuencias en porcentajes para las tres categorías de la variable jugador que centra. Podemos observar que los que más centraron son los centrocampistas $(45,1 \%)$, seguidos de los defensas $(42,6 \%)$. Sobre el porcentaje de casos de los centros realizados por los defensas, podemos observar a su vez, como el $51,4 \%$ de los centros se realizaron desde zona 6 mientras que el $48,6 \%$ se realizaron desde zona 5 .

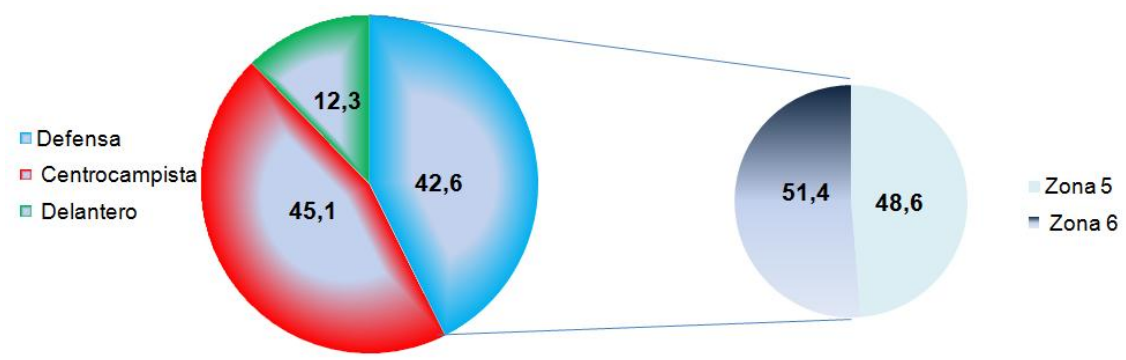

Figura 10: 10A (izquierda) Porcentaje de casos para la variable Jugador que realiza el centro $(N=657)$ y $10 \mathrm{~B}$ (derecha), porcentaje de casos para la variable Zonas del campo donde son realizados los centros de los defensas $\mathrm{N}=(280)$ 


\begin{tabular}{|c|c|c|c|}
\hline \multicolumn{4}{|c|}{$\begin{array}{c}\text { Tabla 4: relación del jugador que centra con la } \\
\text { profundidad }\end{array}$} \\
\hline \multirow{2}{*}{ Jugador que centra } & Frecuencia & Porcentaje \\
\hline \multirow{2}{*}{ Defensa } & Zona 5 & 136 & 48,6 \\
\cline { 2 - 4 } & Zona 6 & 144 & 51,4 \\
\hline \multirow{2}{*}{ Centrocampista } & Zona 5 & 119 & 40,2 \\
\cline { 2 - 4 } & Zona 6 & 177 & 59,8 \\
\hline \multirow{2}{*}{ Delantero } & Zona 5 & 16 & 19,8 \\
\cline { 2 - 4 } & Zona 6 & 65 & 80,2 \\
\hline
\end{tabular}

\subsection{Variables previas al centro por banda.}

Siguiendo con el proceso temporal por el cual ocurren las variables estudiadas que dieron lugar al centro por banda, observamos que, el tipo de ataque posicional, ocurrió el doble de veces $(67,3 \%)$ que tras un robo de balón, con la consecuente transición rápida para el centro $(32,7 \%)$ como podemos observar en la figura 11.

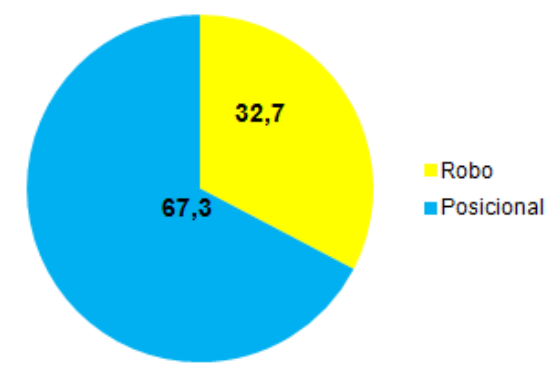

Figura 11: Porcentaje de casos para la variable Tipo de ataque

En la figura 12, observamos el tipo de posesión que fue empleada dentro de los tipos de ataques. El que más se dio con un $49,2 \%$ fue una posesión media (entre 3 y 5 pases) y la circulación realizada por el balón fue homogénea en todas las categorías exceptuando cuando no se dio circulación (8,8\%), como representa la figura 13.
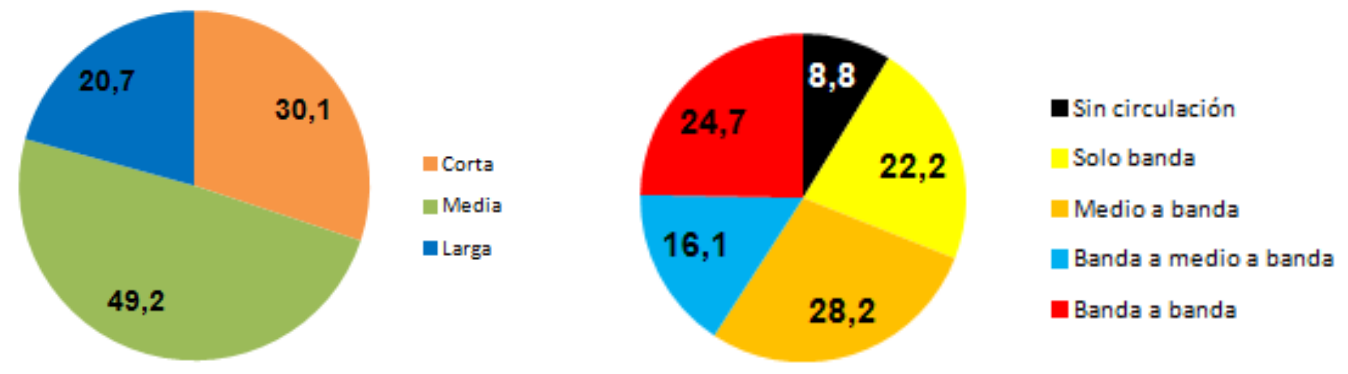

Figura 12: Porcentaje de casos para la variable Posesión categorizada $\mathrm{N}=(657$
Figura 13: Porcentaje de casos para la variable Circulación de balón $\mathrm{N}=(657)$ 
Por último, podemos observar en las figuras $14,15,16$ las variables relativas al último pase previo al centro, la situación espacial en la que se encontraba el centrador y si en ese momento existió o no desdoblamiento. Pues bien, la variable último pase se dio en un $50,4 \%$ de los casos al espacio. Este último pase, aportó en un $66,7 \%$ una situación de ventaja espacial, en la que el rival se encontraba a mas de 3 metros en el momento del centro, justo en el cual, solo en el 34,6\% existió desdoblamiento del lateral.

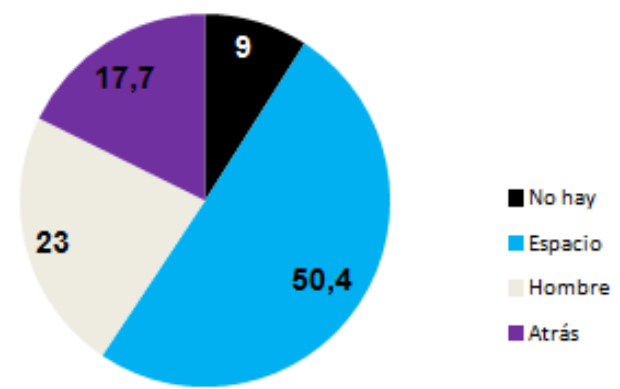

Figura 14: Porcentaje de casos para la variable Último pase $\mathrm{N}=(657)$

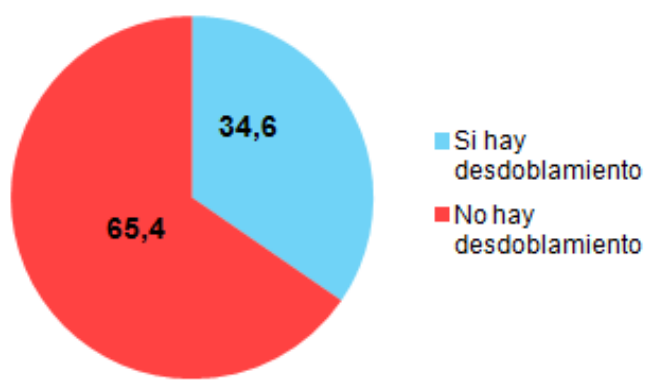

Figura 16: Porcentaje de casos para la variable Desdoblamiento $\mathrm{N}=(657)$

\subsection{Variables durante y posteriores al centro}

En la tabla 5 podemos observar la media y la desviación típica de las variables que se producen durante el centro hasta justo antes de que se contacte con el balón. Cabe destacar que la distancia media que recorre el balón es de 26,712 metros a una velocidad de $65,35 \mathrm{~km} / \mathrm{h}$.

\begin{tabular}{|c|c|c|}
\hline \multicolumn{2}{|c|}{$\begin{array}{c}\text { Tabla 5: Variables durante y posteriores } \\
\text { al centro por banda }\end{array}$} \\
\hline Variable & Media & Desv. típ. \\
\hline Longitud & 26,712 & 9,685 \\
\hline Velocidad & 65,352 & 18,1768 \\
\hline
\end{tabular}


En la figura 17, se puede observar la distribución del número de jugadores totales, compañeros y rivales dentro del área en el momento de contacto con el balón. Se produce un ratio de 1:2 jugadores dentro del área siendo el doble de rivales que el de compañero para rematar el balón.

\section{Número de Jugadores dentro del área}

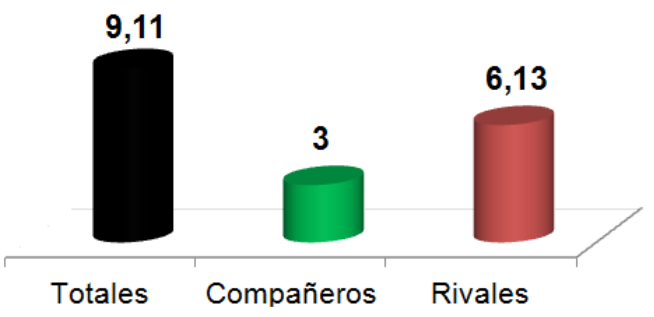

Figura 17: Media de casos para las variables

Totales dentro del área, Compañeros dentro

del área y Rivales dentro del área $\mathrm{N}=(657)$

En la tabla 6, se analizó la relación existente entre el rendimiento del centro con el tipo de ángulo. Se observó una tendencia a mejorar el rendimiento del mismo conforme aumentaba la amplitud del mismo pasando de un $16,4 \%$ en ángulos estrechos a un $18,5 \%$ en ángulos medios a, por ultimo un $22,3 \%$ en ángulos amplios.

\begin{tabular}{|c|c|c|}
\hline $\begin{array}{r}\text { Tabla 6: relación del rendimiento con } \\
\text { el tipo de ángulo. }\end{array}$ \\
\hline \multicolumn{3}{|c|}{ Angulo Estrecho } \\
\hline Rendimiento & Frecuencia & Porcentaje \\
\hline Éxito & 28 & 16,4 \\
\hline No éxito & 143 & 83,6 \\
\hline \multicolumn{3}{|c|}{ Angulo Medio } \\
\hline Éxito & 56 & 18,5 \\
\hline No éxito & 246 & 81,5 \\
\hline \multicolumn{3}{|c|}{ Angulo Amplio } \\
\hline Éxito & 41 & 22,3 \\
\hline No éxito & 143 & 77,7 \\
\hline
\end{tabular}




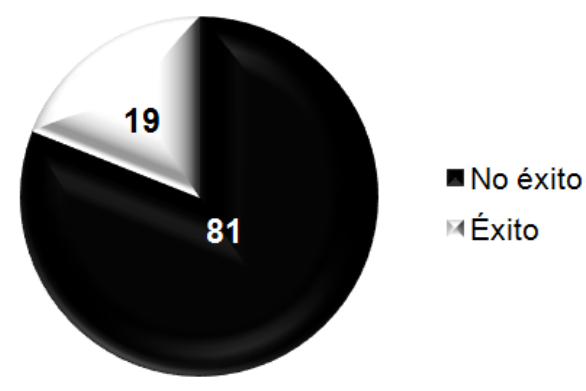

Figura 18: Porcentaje de casos

para la variable Rendimiento

Una vez analizado el rendimiento que aportan los centros por banda (figura 18), podemos observar que el $81 \%$ de los centros no tienen éxito, mientras que el $19 \%$ de las ocasiones son acciones de éxito, ya sea contactando el compañero o marcando gol.

También se realizó una estadística inferencial como podemos observar en la tabla 7 en la que buscamos la asociación entre las diferentes variables estudiadas y el rendimiento, mediante la Rho de Spearman, pero ninguna de ellas alcanzó los niveles de significación y la intensidad del coeficiente de correlación fue nulo.

\begin{tabular}{|c|c|c|c|c|c|c|c|}
\hline \multicolumn{8}{|c|}{$\begin{array}{l}\text { Tabla 7: Valores de } \mathbf{P} \text { y Rho de Spearman para el rendimiento con las variables } \\
\text { circulación de balón, último pase, resultado y tipo de ataque }\end{array}$} \\
\hline & & & Rendimiento & $\begin{array}{l}\text { Circulación } \\
\text { de balón }\end{array}$ & Ultimo pase & Resultado & $\begin{array}{l}\text { Tipo de } \\
\text { ataque }\end{array}$ \\
\hline \multirow{3}{*}{$\begin{array}{c}\text { Rho de } \\
\text { Spearman }\end{array}$} & \multirow{3}{*}{ Rendimiento } & $\begin{array}{l}\text { Coeficiente } \\
\text { de } \\
\text { correlación }\end{array}$ & 1,000 &, 021 &,- 054 &,- 053 &, 017 \\
\hline & & $\begin{array}{c}\text { Sig. } \\
\text { (bilateral) }\end{array}$ & &, 584 &, 167 & ,177 & ,658 \\
\hline & & $\mathrm{N}$ & 657 & 657 & 657 & 657 & 657 \\
\hline
\end{tabular}




\section{Discusión}

Como hemos observado en los resultados del análisis de los centros de equipos de la Liga Española y de la Premier League, los equipos locales realizan más centros $(62,4 \%)$ que los visitantes, lo que resulta ciertamente ventajoso que por el hecho de ser local, tendremos más oportunidades de hacer gol que el equipo visitante en cuanto a centros. Este aspecto de ser local con respecto a ser visitante se ha confirmado en otras investigaciones relacionadas con el comportamiento atacante de los locales y los visitantes como la de Sasaki et al. (1999) y Tucker et al. (2005). Difiere de investigaciones como la de Taylor et al. (2008) que en su muestra de equipos ingleses exclusivamente, no encontró diferencias influenciadas por la condición de local o visitante.

Para deportes de baja puntuación como el futbol, solo hay tres niveles principales sobre el estado del partido: ir ganando, empatando o perdiendo (LagoPeñas, 2012). Se ha demostrado que los jugadores de fútbol, realizan significativamente menos actividad de alta intensidad cuando ganan que cuando pierden o empatan ( $\mathrm{J}$ Castellano et al., 2011). Es posible que al ir ganando, los jugadores, adopten estrategias de juego diferentes como muestra el estudio de Gómez, Gómez-Lopez, Lago, and Sampaio (2012), nos dicen que los equipos que van ganando, exhiben diferentes perfiles de juego a los que van empatando o perdiendo en cuanto a la capacidad de recuperar el balón y sobre la mayor capacidad de generar acciones ofensivas. Nosotros en nuestro estudio no hemos encontrado diferencias sobre lo que influye el resultado del partido en la capacidad de centrar, ya que perdiendo realizaron el $31,2 \%$ y ganando, el $25,4 \%$, en la línea de estudios como Moraes, Cardoso, and Teoldo (2014) quienes también observaron un comportamiento coherente y equilibrado sobre otras variables como los pases realizados y los patrones de recuperación de la pelota en las situaciones de ir ganando empatando o perdiendo, en la Selección Española durante la Copa del Mundo de 2010.

En la actualidad existe una mayor presencia en ataque, de los jugadores atrasados como son los laterales, como bien observamos en nuestro estudio, realizaron el $42,6 \%$ de los centros repartiéndose este porcentaje en la última y penúltima zona del ataque. Este argumento va en línea con otras investigaciones como la de Mahoney, Wheeler, and Lyons (2012) quienes en su estudio demostraron que la probabilidad de marcar gol aumentaba cuando las posesiones finalizaban en las dos últimas zonas del ataque y también cuando se robaba el balón en las mismas. Creemos que una tendencia en el modelo de juego de los equipos profesionales, puede ser el incorporar un mayor número de veces a sus laterales para llegar a las ultimas zonas del ataque, así realizar un mayor número de centros y a su vez estar cercanos de la jugada para recuperar en las ultimas zonas del campo. 
Como ya hemos comentado anteriormente hay estudios que demuestran que los equipos que van perdiendo, aumentan sus niveles de posesión, (James, Jones, \& Mellalieu, 2004; Sasaki et al., 1999) cuyos resultados muestran que las posesiones están condicionadas por la cercanía a la línea de gol. Otras investigaciones (Vogelbein, Nopp, \& Hökelmann, 2014) ponen de relieve la importancia de la recuperación rápida de la posesión del balón. Contrastándolo con nuestro resultados podemos indicar que las transiciones ataque-defensa deben de ser rápidas para intentar hacernos de nuevo con el balón lo antes posible, pero en cuanto a las transiciones defensa-ataque, no tenemos que precipitarnos puesto que en nuestro estudio, observamos que el número de centros realizados en ataque posicional con respecto al robo es el doble $67,3 \%$ y $32,7 \%$ respectivamente, debido a lo cual observamos la necesidad que existe de utilizar posesiones más largas para construir ataques.

Por otra parte, hemos revisado varios artículos que estudiaban las posesiones como Mitschke and Milani (2014) que estudió la técnica para realizar un pase más utilizada en los partidos de futbol de la Eurocopa de 2012 fue el interior. Otros estudios hablan sobre que los mejor equipos, tienden a utilizar estrategias de conservación de la pelota para controlar a sus oponentes, (Collet, 2013; Lago-Peñas \& Dellal, 2010; Lago, 2009; Taylor, Mellalieu, \& James, 2005). Las posesiones también han sido relacionadas con el rendimiento y con los esfuerzos realizados durante el partido (Morgans, Adams, Mullen, \& Williams, 2014), pero no hemos encontrado ningún estudio que nos mostrase los tipos de posesiones en función al número de pases, clasificándolas como hemos realizado nosotros en nuestro estudio, en posesiones cortas (0-2 pases), medias ( 2 a 5 pases) o largas (más de 6) haciendo un clúster con todas las posesiones registradas. Vemos la necesidad de seguir estudiando este tema y ampliar esta variable a un estudio de posesiones sobre todas las acciones del partido para establecer un lenguaje común sobre los tipos de posesiones ya que en nuestro estudio solo estudiamos las previas al centro.

Los resultados de la variable circulación de balón, fueron homogéneos en todas sus categorías (alrededor del $20 \%$ ) a excepción de cuando no hubo circulación de balón con un $8,8 \%$. Incidimos en las categorías en las que el balón circula por un único carril ( $31 \%$ de los casos) con respecto a los casos en los que el balón circula por varios carriles (69\%). Nos lleva a pensar que el doble de veces previo a un centro, hay una circulación de balón por los diferentes carriles del campo, unido a lo comentado anteriormente sobre los tipos de posesiones y los tipos de ataque, entendemos que para obtener un mayor número de centros debemos construir la jugada realizando ataques posicionales y utilizando las bandas para hacerle daño al equipo rival. En la línea que comentamos de amplitud, Clemente, Couceiro, Martins, Mendes, and Figueiredo (2013) en su estudio sobre la dispersión del equipo cuando tenía la posesión del balón, sugirieron una mayor dispersión durante la primera parte en los momentos con mayor posesión, esta mayor dispersión podría ser asociada 
con los principios de juego de anchura y profundidad y los valores de dispersión más bajos se asociaron con principios defensivos del juego así como de recuperación activa del balón.

Además, con respecto a la variable del último pase, hemos podido comprobar cómo el pase al espacio es el más utilizado con el $50,4 \%$ de los casos con respecto a las otras modalidades, lo que nos hace pensar en la necesidad de construir un modelo de juego en el que para aprovechar el juego por banda y sacarle un mayor rendimiento al centro. Debemos dejar esos espacios laterales que sean ocupados de forma sorpresiva por uno de nuestros jugadores. Lamentablemente, este es un aspecto que no hemos podido contrastar con el resto de literatura científica puesto que es una variable tan concreta que no hemos encontrado otros precedentes. Este estudio puede servir para sentar las bases de futuras investigaciones que profundicen mas es aspectos concretos del juego

Las relaciones espacio-temporales especificas entre los jugadores de fútbol, son algunos de los principales factores para la comprensión del juego en su conjunto, sin embargo pocos son los estudios que se han realizado con el fin de analizar los factores que influyen en esas relaciones (Clemente et al., 2013). De lo poco que se ha estudiado sobre las relaciones espacio temporales ha sido conocer la dinámica del uso estratégico del espacio de los equipos que disputan un partido (Julen Castellano, Álvarez-Pastor, \& Blanco-Villaseñor, 2013) o que a pesar de que el terreno de juego es más largo que ancho, los equipos utilizan disposiciones de mayor amplitud que de profundidad (Fradua et al., 2013). En nuestro estudio hemos incidido en el momento espacio temporal del jugador que realiza el centro, observando así que el $66,7 \%$ de las veces el centro lo realiza en una situación en la que el oponente se encuentra a mas de 3 metros de distancia, lo cual puede venir explicado por la calidad de los jugadores del alto nivel, por lo que los rivales están más preocupados de tapar las zonas centrales del juego que los laterales cuando se encuentran en las ultimas zonas de finalización.

Para la variable del desdoblamiento, hemos observado que en el $65,4 \%$ de los casos no se ha producido, por lo que no se muestra como habilidad colectiva básica protagonista en la obtención de un mayor número de centros como podría resultar el tópico entre entrenadores sobre esta habilidad. Creemos que sería necesario un estudio sobre esta habilidad para extraer lo que realmente sucede cuando ocurre.

Unos de los aspectos descriptivos que podemos resumir con nuestro estudio y que arroja una primera visión global sobre los centros y que no se ha realizado antes, es la distancia media que suelen recorrer (26,7 metros) y la velocidad media a la que lo hacen $(65,35 \mathrm{~km} / \mathrm{h})$, lo cual no da una idea a los entrenadores sobre las 
situaciones que deberíamos recrear con algo más de frecuencia cuando queramos trabajar los centros por banda.

También hemos observado que habitualmente hay una media de 9 jugadores en la zona de remate, siendo de estos, el doble rivales que compañeros. No hemos encontrado en la literatura nada semejante, pero consideramos interesante entender que existe un ratio medio de 1:2 como hemos comprobado. Consideramos importante esta variable para los entrenadores, ya que podría tener bastante importancia en el diseño de tareas.

En cuanto a la relación entre el tipo de ángulo utilizado en el centro y el rendimiento hemos observado una tendencia que indica que los centros que mayor éxito producen son los más amplios, lo cual contradice uno de los tópicos de los entrenadores, pensaban que era al contrario, que los más difíciles de defender, eran los de ángulo estrecho ya que el defensor veía reducido su campo de visión. No obstante, hay que ser cautos y sería conveniente realizar un nuevo estudio sobre los ángulos, aumentando la muestra para ver si se confirma la tendencia.

\section{Conclusiones}

1. Los datos muestran una frecuencia claramente superior de centros realizados por los equipos locales.

2. Los jugadores que juegan en líneas atrasadas, como son los laterales, muestran una altísima participación en los centros realizados por los equipos profesionales.

3. No se observan diferencias en el número de centros realizados entre la última zona horizontal de ataque y la penúltima.

4. Los centros realizados por los equipos profesionales en ataque posicional duplican porcentualmente a los centros realizados tras robo, en fase de transición.

5. Los pases al espacio son muy superiores en número al resto de pases preparatorios para realizar los centros. Importante información para la elección de modelo de juego y para el diseño de tareas de entrenamiento.

6. El análisis también nos permite observar que la ventaja espacial para centrar es un comportamiento relevante para realizar un mayor número de centros en comparación a las situaciones sin ventaja.

7. El ratio atacantes-defensores en zona de remate es de 1:2 


\section{Aplicaciones prácticas}

Los campos de aplicación son fundamentalmente dos: la elección de comportamientos adecuados en el modelo de juego y el diseño de tareas de entrenamiento. Como el primero ya ha sido comentado en algunos casos en la discusión, en este apartado nos centraremos en el diseño de tareas de entrenamiento.

A continuación exponemos las aplicaciones prácticas más interesantes en relación al diseño de tareas de entrenamiento respecto a la información obtenida en el estudio.

En relación a los jugadores que juegan en líneas atrasadas, han mostrando una altísima participación en los centros realizados por los equipos profesionales. Este dato debe ser recogido por los entrenadores de alto nivel para que en sus entrenamientos, propongan tareas en las que la incorporación de los laterales sea frecuente, sin diferencias, tanto hasta el último, como al penúltimo sector del campo. Estas variables junto a amplitud de los ángulos, si las relacionamos, puede ser aplicable en tareas que se centren desde la penúltima zona de ataque de igual manera por los defensas o los centrocampistas.

En base a nuestros resultados, los entrenadores pueden ahora desarrollar ejercicios en los que a partir de una circulación de balón, utilicen el ataque posicional, para obtener un mayor rendimiento en los ataques. En cuanto al pase al espacio, y la situación espacial, son variables que aplicando una serie de normas en las tareas, podemos acercarnos a la realidad del juego.

Otro elemento muy interesante que hemos podido extraer de nuestros resultados para el diseño de tareas ha sido el ratio de jugadores dentro del área en el momento de contactar con balón. Este ratio de 1 atacante por cada 2 rivales, nos permitirá utilizar algunas normas con las acercarnos más a la realidad del juego con nuestras tareas. 


\section{Agradecimientos}

Al Ministerio de Educación, Cultura y Deporte, por la Beca para poder realizar el Máster de Investigación en Actividad Física y Deporte junto con el resto de mis estudios.

A Luis, por confiar en todo momento en mis aptitudes, por su apoyo, energía y todo el tiempo invertido en guiarme hacia la consecución de mis propósitos.

A Javi, por estar siempre disponible para las dudas, consultas o material que fuera necesario. Un compañero admirable en todo momento.

\section{Referencias}

Bangsbo, J., Mohr, M., \& Krustrup, P. (2006). Physical and metabolic demands of training and match-play in the elite football player. Journal of Sports Sciences, 24(07), 665674.

Bartlett, R., Button, C., Robins, M., Dutt-Mazumder, A., \& Kennedy, G. (2012). Analysing team coordination patterns from player movement trajectories in soccer: methodological considerations. International Journal of Performance Analysis in Sport, 12(2), 398-424.

Bradley, P. S., Sheldon, W., Wooster, B., Olsen, P., Boanas, P., \& Krustrup, P. (2009). Highintensity running in English FA Premier League soccer matches. Journal of Sports Sciences, 27(2), 159-168.

Carling, C., Bloomfield, J., Nelsen, L., \& Reilly, T. (2008). The role of motion analysis in elite soccer. Sports Medicine, 38(10), 839-862.

Carling, C., Reilly, T., \& Williams, A. M. (2008). Performance assessment for field sports: Routledge.

Carling, C., Williams, A. M., \& Reilly, T. (2005). Handbook of soccer match analysis: A systematic approach to improving performance: Psychology Press.

Castellano, J., Álvarez-Pastor, D., \& Blanco-Villaseñor, Á. (2013). Análisis del espacio de interacción en fútbol. Paper presented at the Revista de psicología del deporte.

Castellano, J., Blanco-Villaseñor, A., \& Alvarez, D. (2011). Contextual variables and timemotion analysis in soccer. International Journal of Sports Medicine, 32(6), 415-421.

Clemente, F. M., Couceiro, M. S., Martins, F. M., Mendes, R., \& Figueiredo, A. J. (2013). Measuring Collective Behaviour in Football Teams: Inspecting the impact of each half of the match on ball possession. International Journal of Performance Analysis in Sport, 13(3), 678-689. 
Cohen, J. (1960). A coefficient of agreement for nominal scales. Educational and psychological measurement, 20(1), 37-46.

Collet, C. (2013). The possession game? A comparative analysis of ball retention and team success in European and international football, 2007-2010. Journal of Sports Sciences, 31(2), 123-136.

Coutts, A. J. (2014). Evolution of football match analysis research. Journal of Sports Sciences, 32(20), 1829-1830.

Drust, B., Atkinson, G., \& Reilly, T. (2007). Future perspectives in the evaluation of the physiological demands of soccer. Sports Medicine, 37(9), 783-805.

Fleiss, J. L., Levin, B., \& Paik, M. C. (2013). Statistical methods for rates and proportions: John Wiley \& Sons.

Fradua, L., Zubillaga, A., Caro, Ó., Iván Fernández-García, Á., Ruiz-Ruiz, C., \& Tenga, A. (2013). Designing small-sided games for training tactical aspects in soccer: Extrapolating pitch sizes from full-size professional matches. Journal of Sports Sciences, 31(6), 573-581.

Gómez, M. A., Gómez-Lopez, M., Lago, C., \& Sampaio, J. (2012). Effects of game location and final outcome on game-related statistics in each zone of the pitch in professional football. European Journal of Sport Science, 12(5), 393-398.

Grant, A., Williams, A., Reilly, T., \& Borrie, T. (1999). Analysis of the goals scored in the 1998 World Cup. Journal of Sports Sciences, 17(10), 826-827.

Griffiths, D. (1999). An analysis of France and their opponents at the 1998 soccer World Cup with specific reference to playing patterns. Unpublished master" s thesis, University of Wales Institute, Cardiff, Wales.

Hughes, M., Churchill, S., Reilly, T., Cabri, J., \& Araújo, D. (2005). Attacking profiles of successful and unsuccessful teams in Copa America 2001. Paper presented at the Science and football V: The proceedings of the fifth world congress on science and football.

Hughes, M., \& Franks, I. (2004). Notational analysis of sport 2nd Edition-a perspective on improving coaching. London: E. \& FN Spon.

Hughes, M., \& Franks, I. (2005). Analysis of passing sequences, shots and goals in soccer. Journal of Sports Sciences, 23(5), 509-514.

Hughes, M., \& Franks, I. M. (2004). Notational analysis of sport: Systems for better coaching and performance in sport: Psychology Press.

Hughes, M., Robertson, K., \& Nicholson, A. (1988). Comparison of patterns of play of successful and unsuccessful teams in the 1986 World Cup for soccer. Science and football, 363-367. 
Hughes, M. D., \& Bartlett, R. M. (2002). The use of performance indicators in performance analysis. Journal of Sports Sciences, 20(10), 739-754.

James, N., Jones, P., \& Mellalieu, S. (2004). Possession as a performance indicator in soccer as a function of successful and unsuccessful teams. Journal of Sports Science, 22(6), 507-508.

Jones, N. M., Mellalieu, S. D., \& James, N. (2004). Team performance indicators as a function of winning and losing in rugby union. International Journal of Performance Analysis in Sport, 4(1), 61-71.

Lago-Ballesteros, J., \& Lago-Peñas, C. (2010). Performance in team sports: Identifying the keys to success in soccer. Journal of Human Kinetics, 25, 85-91.

Lago-Peñas, C. (2012). The role of situational variables in analysing physical performance in soccer. Journal of Human Kinetics, 35(1), 89-95.

Lago-Peñas, C., \& Dellal, A. (2010). Ball possession strategies in elite soccer according to the evolution of the match-score: the influence of situational variables. Journal of Human Kinetics, 25, 93-100.

Lago, C. (2009). The influence of match location, quality of opposition, and match status on possession strategies in professional association football. Journal of Sports Sciences, 27(13), 1463-1469.

Lago, C., Casais, L., Dominguez, E., \& Sampaio, J. (2010). The effects of situational variables on distance covered at various speeds in elite soccer. European Journal of Sport Science, 10(2), 103-109.

Liebermann, D. G., Katz, L., Hughes, M. D., Bartlett, R. M., McClements, J., \& Franks, I. M. (2002). Advances in the application of information technology to sport performance. Journal of Sports Sciences, 20(10), 755-769.

Mahoney, L. E., Wheeler, K. W., \& Lyons, K. (2012). Analysis of Factors Determining Invasion into Attacking Areas and the Creation of Goal-Scoring Opportunities in the Asian Cup Football Competition. Asian Journal of Exercise and Sports Science, 9(1).

Mitschke, C., \& Milani, T. (2014). Soccer: Detailed Analysis of Played Passes in the UEFA Euro 2012. International Journal of Sports Science and Coaching, 9(5), 1019-1032.

Mohr, M., Krustrup, P., \& Bangsbo, J. (2003). Match performance of high-standard soccer players with special reference to development of fatigue. Journal of Sports Sciences, 21(7), 519-528.

Moraes, E. L., Cardoso, F., \& Teoldo, I. (2014). Análise dos padrões ofensivos da Seleção Espanhola de Futebol na Copa do Mundo FIFA® 2010 em relação ao "status" da partida. Revista Brasileira de Educação Física e Esporte, 28(3), 361-369. 
Morgans, R., Adams, D., Mullen, R., \& Williams, M. D. (2014). Changes in physical performance variables in an English Championship League team across the competitive season: the effect of possession. International Journal of Performance Analysis in Sport, 14(2), 493-503.

Nevill, A., Atkinson, G., \& Hughes, M. (2008). Twenty-five years of sport performance research in the Journal of Sports Sciences. Journal of Sports Sciences, 26(4), 413-426.

O'Donoghue, P. (2005). Normative profiles of sports performance. International Journal of Performance Analysis in Sport, 5(1), 104-119.

Rampinini, E., Coutts, A., Castagna, C., Sassi, R., \& Impellizzeri, F. (2007). Variation in top level soccer match performance. International Journal of Sports Medicine(28), 10181024.

Reilly, T., \& Thomas, V. (1976). A motion analysis of work-rate in different positional roles in professional football match-play. Journal of human movement studies, 2(2), 87-97.

Sarmento, H., Marcelino, R., Anguera, M. T., CampaniÇo, J., Matos, N., \& LeitÃo, J. C. (2014). Match analysis in football: a systematic review. Journal of Sports Sciences, $32(20), 1831-1843$.

Sasaki, Y., Nevill, A., \& Reilly, T. (1999). Home advantage: A case study of Ipswich Town football club during the 1996-1997 season. Journal of Sports Sciences, 17, 831.

Stanhope, J. (2001). An investigation into possession with respect to time, in the soccer world cup 1994. Notational Analysis of Sport III. Cardiff: Centre for Performance Analysis, UWIC, 155-162.

Tabachnick, B. G., \& Fidell, L. S. (2013). Using multivariate statistics (Boston Ed. 6th ed.).

Taylor, J. B., Mellalieu, S. D., \& James, N. (2005). A comparison of individual and unit tactical behaviour and team strategy in professional soccer. International Journal of Performance Analysis in Sport, 5(2), 87-101.

Taylor, J. B., Mellalieu, S. D., James, N., \& Shearer, D. A. (2008). The influence of match location, quality of opposition, and match status on technical performance in professional association football. Journal of Sports Sciences, 26(9), 885-895.

Tenga, A., Holme, I., Ronglan, L. T., \& Bahr, R. (2010). Effect of playing tactics on goal scoring in Norwegian professional soccer. Journal of Sports Sciences, 28(3), 237-244.

Tucker, W., Mellalieu, S. D., James, N., \& Taylor, J. B. (2005). Game location effects in professional soccer: A case study. International Journal of Performance Analysis in Sport, 5(2), 23-35.

Valter, D. S., Adam, C., Barry, M., \& Marco, C. (2006). Validation of Prozone®: A new video-based performance analysis system. International Journal of Performance Analysis in Sport, 6(1), 108-119. 
Vogelbein, M., Nopp, S., \& Hökelmann, A. (2014). Defensive transition in soccer-are prompt possession regains a measure of success? A quantitative analysis of German FußballBundesliga 2010/2011. Journal of Sports Sciences, 32(11), 1076-1083.

Zubillaga, A., Gorospe, G., Hernandez Mendo, A., \& Villasenor, A. (2007). Analysis of high intensity activity in soccer highest level competition. J Sports Sci Med, 6(Suppl 10), $10-11$. 